\title{
Permeability of a growing biofilm in a porous media fluid flow analyzed by magnetic resonance displacement-relaxation correlations
}

\section{Authors: Sarah J. Vogt, Alexis B. Sanderlin, Joseph D. Seymour, \& Sarah L. Codd}

NOTICE: This is the peer reviewed version of the following article: Vogt SJ, Sanderlin AB, Seymour JD, Codd SL, "Permeability of a growing biofilm in a porous media fluid flow analyzed by magnetic resonance displacement-relaxation correlations," Biotechnology and Bioengineering, May 2013 110(5):1366-1375, which has been published in final form at http://dx.doi.org/10.1002/ bit.24803. This article may be used for non-commercial purposes in accordance with Wiley Terms and Conditions for Self-Archiving.

Vogt SJ, Sanderlin AB, Seymour JD, Codd SL, "Permeability of a growing biofilm in a porous media fluid flow analyzed by magnetic resonance displacement-relaxation correlations," Biotechnology and Bioengineering, May 2013 110(5):1366-1375. 


\title{
Permeability of a Growing Biofilm in a Porous Media Fluid Flow Analyzed by Magnetic Resonance Displacement- Relaxation Correlations
}

\author{
Sarah J. Vogt, ${ }^{1,2}$ Alexis B. Sanderlin, ${ }^{1,2}$ Joseph D. Seymour, ${ }^{1,2}$ Sarah L. \\ $\operatorname{Codd}^{2,3}$ \\ 1 \\ Department of Chemical and Biological Engineering, Montana State University, \\ Bozeman, Montana \\ ${ }_{2}$ Center for Biofilm Engineering, Montana State University, Bozeman, Montana Department of Mechanical and Industrial \\ Engineering, Montana State University, \\ 3220 Roberts Hall, PO Box 173800, Bozeman, Montana 59717
}

\section{ABSTRACT:}

Biofilm growth in porous media is difficult to study non-invasively due to the opaqueness and hetero-geneity of the systems. Magnetic resonance is utilized to non-invasively study water dynamics within porous media. Displacement-relaxation correlation experiments were per-formed on fluid flow during biofilm growth in a model porous media of mono-dispersed polystyrene beads. The spin-spin $\mathrm{T} 2$ magnetic relaxation distinguishes between the biofilm phase and bulk fluid phase due to water-biopolymer interactions present in the biofilm, and the flow dynamics are measured using PGSE NMR experiments. By correlating these two measurements, the effects of biofilm growth on the fluid dynamics can be separated into a detailed analysis of both the biofilm phase and the fluid phase simultaneously within the same experiment. Within the displacement reso-lution of these experiments, no convective flow was mea-sured through the biomass. An increased amount of longitudinal hydrodynamic dispersion indicates increased hydrodynamic mixing due to fluid channeling caused by biofilm growth. The effect of different biofilm growth con-ditions was measured by varying the strength of the bacterial growth medium.

KEYWORDS: biofilm growth; porous media; permeability; NMR; transport; flow

\section{Introduction}

In diverse growth environments, microorganisms including bacteria and algae will attach to surfaces and grow in a phenotypically different state known as a biofilm. Biofilm growth occurs after the microorganisms attach to a surface by excreting and then growing within the extracellular polymeric substance (EPS; Sutherland, 2001). The EPS consists of proteins, polymers, and DNA material and both enables the bacterial colony to adhere to the surface and inhibits the killing of the bacteria by limiting the diffusion of antimicrobials within the polymer matrix and promoting dormant growth states for the microbial cells. When bacterial colonies grow in biofilms, bacteria are difficult to remove and lead to negative consequences such as persistent infections on medical implants (Costerton et al., 1999). Positive attributes such as the growth of biobarriers to contain harmful environmental contaminants such as nuclear waste are also of interest (Beveridge et al., 1997; Fang et al., 2009).

The experimental observation of biofilm growth in porous media is complicated by the heterogeneous nature of the biofilm, the opaqueness and heterogeneity of porous media growth environments, and the diversity of nutrient conditions and colony species compositions. Thus there are a large number of computer models that have been developed to enable variation of parameters such as growth rates and substrate geometries numerically (Klapper et al., 2002). Recent numerical models utilize either computa-tional fluid dynamics simulations (CFD; Radu et al., 2012) or Lattice-Boltzmann simulations (LB) to calculate both the velocity field of the fluid and to model bacterial transport and attachment (Pintelon et al., 2012; von der Schulenburg et al., 2009). In particular, recent modeling research has been focused on understanding the effect of the permeability of 
the biomass on the flow through biofouled media (Kim and Fogler, 2000; Stewart, 2012). Understanding the biofilm permeability is important for analyzing the change in the overall permeability of the biofouled porous media and for analyzing the transport of nutrients or antimicrobials into the biofilm.

CFD and LB simulations numerically solve the NavierStokes equations for incompressible fluid flow:

$$
\begin{gathered}
\frac{\partial \vec{u}}{\partial t}+\vec{u} \cdot \nabla \vec{u}+\frac{1}{\rho} \nabla p=\eta \nabla^{2} \vec{u} \\
\nabla \cdot \vec{u}=0
\end{gathered}
$$

where $\vec{u}$ is the velocity of the fluid element, $\rho$ is the density of the fluid, $p$ is the pressure, and $\eta$ is the dynamic viscosity of the fluid. Nutrient transport and consumption in the biofilm are modeled using a mass transfer convectiondiffusion-reaction equation (von der Schulenburg et al., 2009):

$$
\frac{\partial C_{\mathrm{s}}}{\partial t}+\vec{u} \cdot \nabla C_{\mathrm{s}}-D_{\mathrm{s}} \nabla^{2} C_{\mathrm{s}}=r_{\mathrm{s}}
$$

where $C_{\mathrm{s}}$ is the nutrient concentration, $D_{\mathrm{s}}$ is the nutrient diffusion coefficient, and $r_{\mathrm{s}}$ is the local consumption rate of the nutrient given by various kinetic models, for example, Monod kinetics. The impact of permeability of the biofilm on convection and diffusion of nutrients is therefore important to quantify in order to model biofilm growth kinetics (Pintelon et al., 2012; von der Schulenburg et al., 2009).

Simulations of biofilm growth in porous media have taken different approaches to the modeling of the permeability of the biofilm. Some models (Kapellos et al., 2007; Radu et al., 2012) account for the permeability of the biofilm by incorporating the Brinkman flow equations (Brinkman, 1947) into the model calculations. Brinkman flow equations model the flow through the biofilm by assuming that the biofilm is a dense array of spherical bacteria in a fluid. Radu et al. (2012) include the full equation in their model:

$$
\frac{\eta}{\kappa} \vec{u}+\nabla p=\frac{1}{\varepsilon}\left[\eta \cdot \nabla^{2} \vec{u}-\left(\frac{2 \eta}{3}-\kappa\right)(\nabla \cdot \vec{u})\right]
$$

where $\varepsilon$ and $\kappa$ are the porosity and permeability of the biofilm, respectively. Kapellos et al. (2007) simplify the calculation by combining Equations (1) and (4) and considering the biofilm and fluid as a continuous fluid with spatially varying properties. The use of the Brinkman equations requires the assumption of values for the porosity, permeability, and viscosity of the biofilm and for the bacterial cell size.

Pintelon et al. (2012) take the simpler approach of solving Equations (1) and (2) assuming an increased value of viscosity of the biofilm in relation to the bulk fluid (Dupin et al., 2001; Thullner and Baveye, 2008). The authors found the predicted relationship between the permeability of the biomass and the velocity of fluid through the biofilm, the growth rate of the biofilm, and the transport of nutrients into the biomass (Pintelon et al., 2012). They demonstrated that the value for the permeability of the biomass significantly affected both the properties of the fluid flow throughout the porous medium and growth rate of the biofilm due to the varying amount of nutrient transport into the biofilm (Pintelon et al., 2012).

The amount of convective flow through the biofilm in a biofouled porous media is currently an open question. Due to the heterogeneity and the different length and time scales for fluid flow through the biofilm and in the bulk, experimental data for realistic systems is difficult to obtain. The work presented here utilizes a recently developed magnetic resonance (MR) experimental technique to study these systems.

\section{Magnetic Resonance}

Magnetic resonance experimental techniques such as spectroscopy (Hornemann et al., 2008; McLean et al., 2008), imaging (Bartacek et al., 2009; Hoskins et al., 1999; Manz et al., 2003; Renslow et al., 2010; Seymour et al., 2004a), and dynamic measurements (Seymour et al., 2004b; von der Schulenburg et al., 2008a,b) have found increasing use in recent years as a non-invasive way to study biofilm growth. The work presented here uses the correlation between molecular motion and MR relaxation to study the growth of a biofilm. These displacement-relaxation correlation experiments (Callaghan, 2011) have been utilized in recent years to study the flow through rocks (Burcaw et al., 2010; Mitchell et al., 2008b; Washburn and Callaghan, 2007), the transport of water through trees (Windt et al., 2007), and water flow through a packed bed of alginate polymer beads (Britton et al., 2004). This technique has been used previously to study a biosystem of packed methanogenic sludge particles (Homan et al., 2010) but to our knowledge has not been used to study convection through a growing biofilm.

Previous studies have utilized dynamic MR measurements to study the transport through biofouled porous media (Pintelon et al., 2010; Seymour et al., 2004b, 2007) but were unable to distinguish between the dynamics within the biomass and in the bulk fluid flow. The biofilm was shown to grow in preferential flow pathways but the degree of flow through the biomass itself was not quantified. MR relaxation measurements have been used to separately study the biofilm and the bulk fluid (Codd et al., 2011) but did not measure the fluid dynamics. The research presented here utilizes these two MR phenomena to study the dynamics within the biomass separately from the bulk fluid at the same time and within the same experiment. By obtaining signal from the entire sample and using the ability to separate the 
biofilm and fluid phases using relaxation phenomena, complicated and heterogeneous biofouled porous media samples may be analyzed.

\section{Measurement of Molecular Motion Using MR}

The MR pulse sequences known as pulsed gradient spin echo (PGSE) and pulsed gradient stimulated echo (PGSTE) are the building blocks for most sequences that measure molecular motion (Callaghan, 2011; Stejskal and Tanner, 1965). These experiments are able to quantify molecular motion by applying a sequence of radio frequency (rf) pulses and magnetic field gradients to encode molecules for their positions at different points in time. The frequency of the precession of the magnetic moments of molecules is encoded for position by applying a linearly varying magnetic field gradient $g$ for a duration $\delta$, a specific waiting time $\Delta$ is then applied during which the molecules move randomly due to diffusion and hydrodynamic dispersion or coherently due to convection, and then the new positions are encoded by applying the same gradient of opposite polarity. The effect of the molecular motion on the phase and amplitude of the total MR signal can then be analyzed. A Fourier relationship is found (Callaghan, 1991, 2011) between the MR signal and the transition probability, or propagator of motion, $P\left(z \mid z^{\prime}, \Delta\right)$ in one direction, defined as the probability that a molecule starting at position $z$ at $t=0$ will move to position $z^{\prime}$ at $t=\Delta$. When we define $Z=z^{\prime}-z$ as the displacement and $q=\frac{\gamma \delta g}{2 \pi}$ as its Fourier conjugate, we can write the signal as:

$$
E(q)=\int P(Z, \Delta) \exp (i 2 \pi q Z) \mathrm{d} Z
$$

where $E(q)$ is the measured MR signal voltage. For example, the propagator for molecular self-diffusion $D$ with a coherent velocity in the $z$ direction is a Gaussian function:

$$
P(Z, \Delta)=\frac{1}{\sqrt{4 \pi D \Delta}} \exp \left[\frac{-(Z-\bar{v} \Delta)^{2}}{4 D \Delta}\right]
$$

By performing a Fourier transform the form for the MR signal is then predicted to be:

$$
E(q)=\exp \left(-4 \pi^{2} q^{2} D \Delta+2 \pi i q \bar{v} \Delta\right)
$$

which demonstrates that diffusion will cause an attenuation of the signal while velocity will result in a phase shift (Callaghan, 1991). The data obtained using the propagator of motion contains information about self-diffusion and flow directly, but also spatial and temporal information in more indirect ways (Callaghan et al., 1999). The resolution in the displacement measurement enables the measurement of both slow flow which may be present through the biofilm and fast flow of the bulk fluid through non-biofouled pores in the same experiment.

\section{MR Relaxation Theory}

The relaxation of the MR signal occurs after an rf pulse excites the molecular magnetic moments and relaxes back to equilibrium due to spin-lattice $T_{1}$ interaction in the longitudinal direction along the applied magnetic field, and spin-spin $T_{2}$ dipolar interactions. The $T_{2}$ relaxation occurs due to both interactions with the other molecules in the solution and also the environment around them. In solution, the $T_{2}$ is dependent on rotational mobility of the proton $\left({ }^{1} \mathrm{H}\right)$ spins (Abragam, 1961), so that on a polymer molecule the $T_{2}$ of the protons is short, on the order of tens to hundreds of $\mathrm{ms}$, but for protons on liquid molecules such as water the $T_{2}$ is long, on the order of seconds. Molecular exchange occurring on a time scale of microsecond or millisecond between polymer protons and water protons will affect the measured $T_{2}$ (Carver and Richards, 1972), resulting in the measured $T_{2}$ being a time and weighted average of the polymer and water proton $T_{2}$ times. For water within the EPS of a biofilm, the molecular exchange between the protons on the polymer molecules and the protons of the water will result in a decrease in the measured $T_{2}$, from a $T_{2}$ of approximately $2 \mathrm{~s}$ in pure water to a $T_{2}$ on the order of $100 \mathrm{~ms}$ in biofilms (Codd et al., 2011; Lens et al., 1997, 1999; Seymour et al., 2004a,b). The displacement-relaxation correlation experiments presented here utilize the capability of MR to quantify both dynamics and molecular relaxation to study the dynamics of the bulk water and the biofilm separately within the same experiment as discussed below.

\section{Materials and Methods}

\section{Biofilm Growth}

Bacillus mojavensis was chosen for these experiments due to the ease with which it produces a relatively thick biofilm and environmental importance (Mitchell et al., 2008a). Brain Heart Infusion (BHI, Becton, Dickenson and Company, Sparks, MD) used as the bacterial growth medium was prepared according to the recipe given by Mitchell et al. (2008a) [40 g/L NaCl, $3 \mathrm{~g} / \mathrm{L} \mathrm{NaNO}_{3}, 0.75 \mathrm{~g} / \mathrm{L}$ $\mathrm{NH}_{4} \mathrm{Cl}$ (Fisher, Pittsburgh, PA), and 28, 18, 9, or $4.5 \mathrm{~g} / \mathrm{L} \mathrm{BHI}$ for $65 \%, 50 \%, 25 \%$, and $12.5 \%$ strength, respectively] and autoclaved. Following preparation and cooling of the media, $1 \mathrm{~mL}$ of a frozen stock culture of the bacteria was warmed to room temperature and added to $100 \mathrm{~mL}$ of $100 \%$ strength BHI. This culture was incubated in a $1 \mathrm{~L}$ media bottle (Kimax, Vineland, NJ) at room temperature on a horizontal shaker for $24 \mathrm{~h}$.

A model porous media was constructed with a $8.76 \mathrm{~mm}$ inner diameter chromatography column (Omnifit, Danbury, CT) that was packed with $240 \mu \mathrm{m}$ diameter monodispersed polystyrene spheres (Duke Scientific, Fremont, CA). The flow loop was constructed using PTFE tubing and HPLC fittings (GE Scientific, Pittsburgh, PA). The bead column was 
saturated with distilled water and was monitored to ensure that there were no leaks or bubbles in the system before being loaded into the MR magnet. The fluid was then switched from water to BHI pumped at a rate of $50 \mathrm{~mL} / \mathrm{h}$ with a HPLC pump (Pharmacia P-500, Thermo Fisher Scientific, Waltham, MA) to both create the proper $\mathrm{pH}$ and osmotic conditions for the bacterium $B$. mojavensis within the column and to perform experiments as a base line before the introduction of the bacteria.

After the bacteria grew sufficiently in the $1 \mathrm{~L}$ media bottle on the shaker, the column was inoculated and incubated for $4 \mathrm{~h}$ to allow for bacterial attachment to the beads. The flow of the growth media (BHI) was then re-started and was continuously pumped through the column at $50 \mathrm{~mL} / \mathrm{h}$ for the duration of the experiments. For the measured initial porosity of $\phi=0.43$, this flow rate gives a superficial velocity $\left\langle v_{0}\right\rangle=0.53 \mathrm{~mm} / \mathrm{s}$ corresponding to a pore Reynolds number $\operatorname{Re}=\frac{\left\langle v_{0}\right\rangle l \rho}{\mu}=9.6 \cdot 10^{-5}$ for water based on a pore length scale $l \stackrel{\mu}{=} \frac{d_{\mathrm{p}} \phi}{1-\phi}=180 \mu \mathrm{m}$, where $d_{\mathrm{p}}=240 \mu \mathrm{m}$ is the diameter of the bead particles. This indicates a Peclet number for the relative contribution of convective to diffusive transport rate of $P e=\frac{\left\langle v_{0}\right\rangle l}{D_{0}}=48$.

The amount of growth media provided for bacteria growth will affect the growth rate of the biofilm through the column. This was varied by using four different strengths of $\mathrm{BHI}, 65 \%, 50 \%, 25 \%$, and $12.5 \%$. For the $65 \%$ and $50 \%$ strength BHI trials, the biofilm grew quickly and plugged the system and caused leaks after $24 \mathrm{~h}$ of data was obtained. The majority of the data discussed in this work was obtained with $25 \%$ and $12.5 \%$ strength BHI which allowed for biofilm growth to be monitored for 2 or 3 days.

\section{Displacement-Relaxation Correlation Experiments}

Two-dimensional $P(Z, \Delta)-T_{2}$ displacement-relaxation correlation experiments were conducted as the biofilm was grown within a $300 \mathrm{MHz}$ Bruker super-wide bore superconducting magnet networked to an AVANCE III spectrometer and a Micro2.5 micro-imaging probe with maximum gradients in all three directions of $1.482 \mathrm{~T} / \mathrm{m}$. $P(Z, \Delta)-T_{2}$ experiments begin with a pulsed gradient, stimulated echo sequence (PGSTE) to encode the spins for displacement followed by a CPMG pulse train (Callaghan, 1991; Carr and Purcell, 1954) to measure the $T_{2}$, as shown in Figure 1 (Callaghan, 2011). A $\tau_{2}$ value of $200 \mu$ s was used to minimize the effects of exchange between $T_{2}$ populations during the $T_{2}$ measurement (Homan et al., 2010). The PGSTE sequence is a variant of the PGSE except that the magnetization is stored along the $z$-axis during the observation time $\Delta$, minimizing the influence of $T_{2}$ relaxation during the observation time $\Delta$ (Callaghan, 1991). Experimental details are discussed in the caption to Figure 1 . The resulting $2 \mathrm{D}$ data set from each experiment included 51 gradient steps in one-dimension and 4,096 echoes in the second dimension. The data was zero-filled to 64 points and a Fourier transform was performed in the

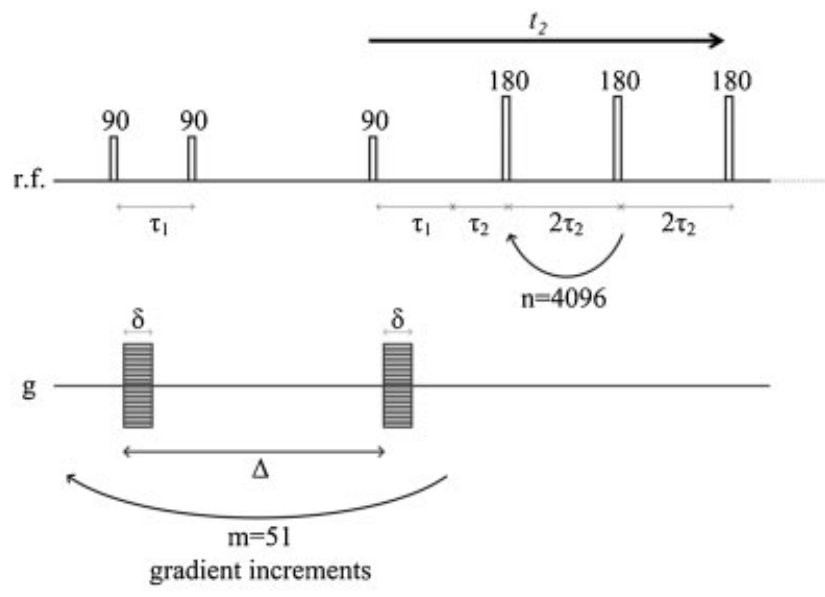

Figure 1. $P(Z, \Delta)-T_{2}$ displacement-relaxation correlation pulse sequence. A PGSTE sequence encoded the spins for displacement before a CPMG sequence was used to measure the $T_{2}$. Experimental parameters: $\delta=1 \mathrm{~ms} ; \Delta=500 \mathrm{~ms}, 250 \mathrm{~ms}$, or $50 \mathrm{~ms}$; 51 linearly spaced gradient increments from $-0.2964 \mathrm{~T} / \mathrm{m}$ to $0.2964 \mathrm{~T} / \mathrm{m}$ for $\Delta=500 \mathrm{~ms},-0.5928 \mathrm{~T} / \mathrm{m}$ to $0.5928 \mathrm{~T} / \mathrm{m}$ for $\Delta=250 \mathrm{~ms}$, and $-1.482 \mathrm{~T} / \mathrm{m}$ to $1.482 \mathrm{~T} / \mathrm{m}$ for $\Delta=50 \mathrm{~ms} ; T_{2}$ from 0 to $1.64 \mathrm{~s}$ with $\tau_{2}=200 \mu \mathrm{s}$ and 4,096 echoes collected.

PGSTE direction to obtain a propagator of motion for each echo that was collected. An inverse Laplace transform (ILT) was then performed for each point in the propagator direction to obtain the spectrum $F\left(P(Z, \Delta), T_{2}\right)$. The ILT was performed using a nonlinear least squares algorithm and:

$$
\begin{aligned}
& M\left(Z, \Delta, t_{2}\right) \\
& =\int P(Z, \Delta) \exp \left(\frac{-t_{2}}{T_{2}}\right) F\left(P(Z, \Delta), T_{2}\right) \mathrm{d} T_{2} \\
& \quad+E\left(Z, t_{2}\right)
\end{aligned}
$$

where $E\left(Z, T_{2}\right)$ represents the noise of the experiment (Venkataramanan et al., 2002). Each experiment was $3 \mathrm{~h}$ in length to allow for signal averaging. Experiments with displacement observation times $\Delta=500,250$, and $50 \mathrm{~ms}$ were performed sequentially as the biofilm was growing within the magnet, resulting in a complete experimental series that required $9 \mathrm{~h}$ to acquire. Biofilms were grown for from 1 to 3 days depending on the growth media strength and the corresponding extent of biofouled clogging of the system.

\section{Results and Discussion}

The data obtained for the biofilm grown using $12.5 \%$ strength BHI growth media is presented in detail and is representative of all the data. At this media strength, the growth reached a pseudo-steady state and was monitored for about 3 days without plugging the system and causing leaks. Figure 2 presents the results of $2 \mathrm{D} P(Z, \Delta)-T_{2}$ correlation experiments for displacement times $\Delta=500$ and $250 \mathrm{~ms}$. 

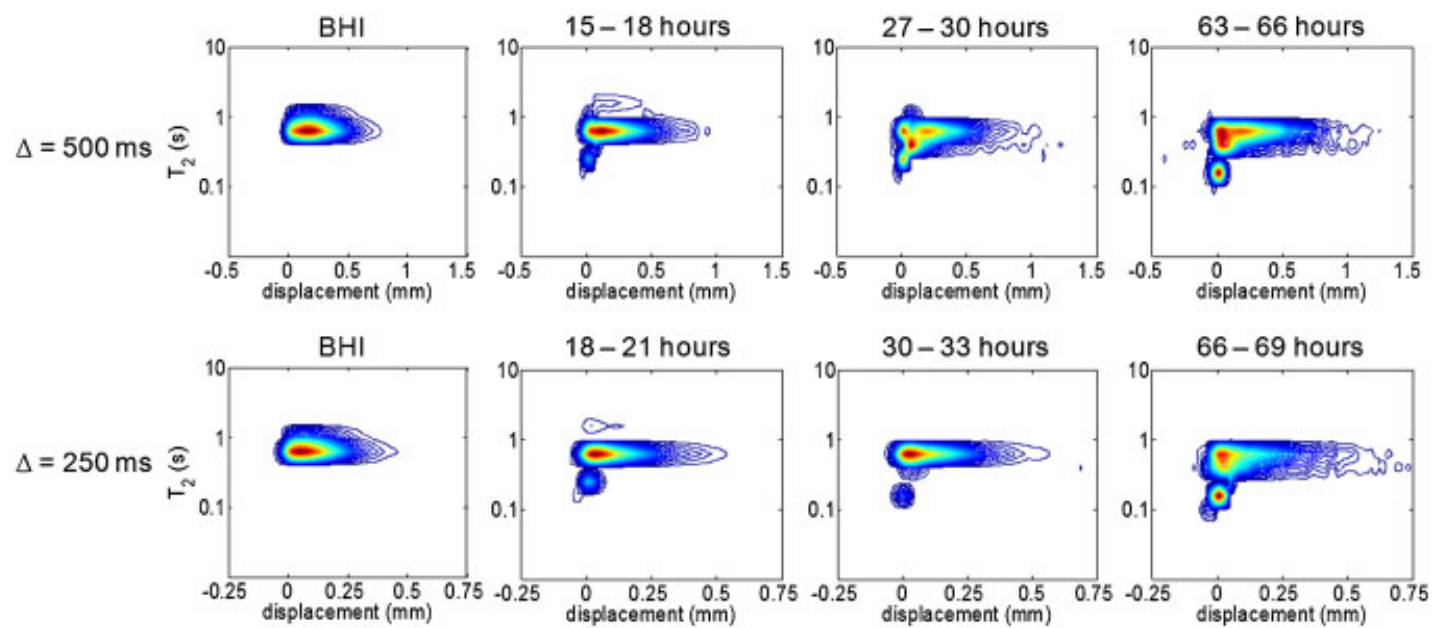

Figure 2. $P(Z, \Delta)-T_{2}$ data as a function of biofilm growth time for $12.5 \%$ strength $B H I$ for observation times $\Delta$ of 250 and $500 \mathrm{~ms}$. The shorter $T_{2}$ peak at $\sim 150 \mathrm{~ms}$ corresponding to biofilm growth increases over time and shows no convective flow. The longer $T_{2}$ peak at $700 \mathrm{~ms}$ corresponding to the bulk fluid exhibits longer displacements as the biofilm grows indicating that there is channeling occurring where the biofilm is plugging up some of the pores and other pores are showing increased velocity due to the fixed volumetric flow rate.

The first column shows the data for BHI growth media flowing through the system before introduction of the bacteria. There is a $T_{2}$ shift to a lower value from distilled water (not shown) from $1.2 \mathrm{~s}$ to $700 \mathrm{~ms}$ due to the carbohydrates and proteins in the BHI, and all of the BHI liquid showed the same $T_{2}$ value. The shape of the curve in the propagator dimension will be discussed further below, note the difference in the displacement axis with longer displacement range for $\Delta=500 \mathrm{~ms}$.

At $t=15-18 \mathrm{~h}$, after the column was inoculated with the bacteria, a shorter $T_{2}$ peak at approximately $150 \mathrm{~ms}$ appears in the distribution and then increases in intensity with increasing biofilm growth time. The maximum and average $T_{2}$ value for this peak varies slightly with biofilm growth time, and thus is referred to in this discussion as $T_{2} \sim 150 \mathrm{~ms}$. The integral of the intensity of the $T_{2} \sim 150 \mathrm{~ms}$ peak over growth time is shown in Figure 3. This peak is located at the $T_{2}$ of the water within the EPS of the biofilm and its amplitude corresponds to the quantity of biofilm in the column. While the peak amplitude tends to increase in amplitude over time, between 30 and $33 \mathrm{~h}$ the peak amplitude decreases before again increasing until $60 \mathrm{~h}$. This corresponds to a sloughing event (Picioreanu et al., 2001) which occurs when part of the biofilm is dispersed due to shear stresses and thus flows out of the bead pack. The distribution of $T_{2}$ values in the data set for $\Delta=500 \mathrm{~ms}$ at

Figure 3. Integral of the intensity of the $T_{2} \sim 150 \mathrm{~ms}$ peak as a function of biofilm growth time for (a) $\Delta=500 \mathrm{~ms}$ and (b) $\Delta=250 \mathrm{~ms}$. The peak was integrated over the same area (from 150 to $250 \mathrm{~ms}$ in the $T_{2}$ direction) of each $P(Z, \Delta)-T_{2}$ plot and then normalized to the total signal intensity of each experiment. Each experimental data point is averaged over a $3 \mathrm{~h}$ growth time period. A sloughing event appears to have occurred after about $30 \mathrm{~h}$ of biofilm growth and is shown by the decrease in signal intensity of the $T_{2}$ component that corresponds to the biomass. The biofilm then continued to grow and the signal intensity increased until after $60 \mathrm{~h}$ when the biofilm appears to have sloughed again. Note the $\Delta=250 \mathrm{~ms}$ data points occur $3 \mathrm{~h}$ after the $\Delta=500 \mathrm{~ms}$ data points.
$33 \mathrm{~h}$ shows an intermediate value for $T_{2}$ between the bulk liquid and the biofilm. This likely corresponds to biomass which detached during the sloughing event and moved through the bead pack. While the experiment acquisition

\section{a $\Delta=500 \mathrm{~ms}$}

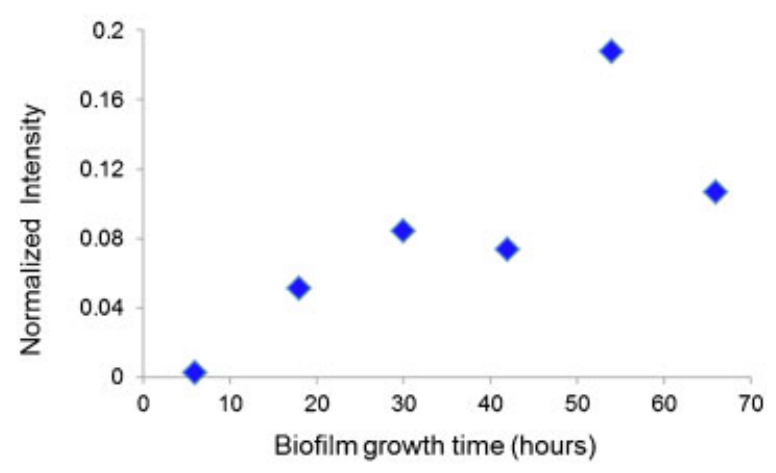

b $\Delta=250 \mathrm{~ms}$

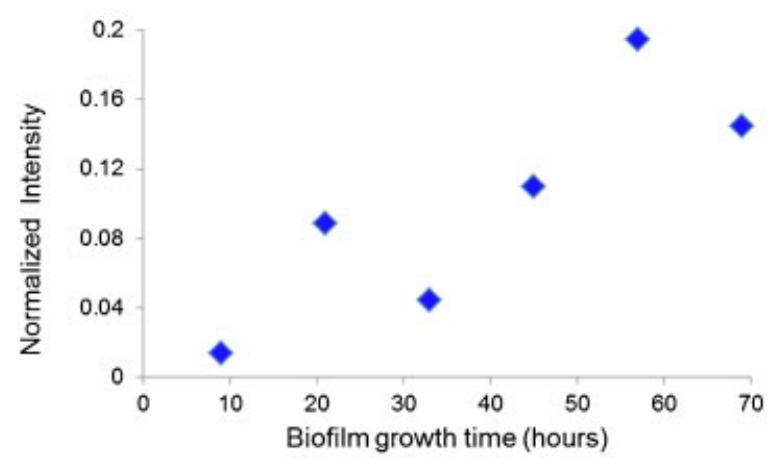


times are too long to pinpoint exactly when the sloughing occurred, there is clearly a transition between 30 and $36 \mathrm{~h}$ in the flow pattern consistent with this interpretation. In addition, the $T_{2}$ distribution shifts to longer values, the shorter $T_{2}$ peak has a smaller intensity, and the bulk flowing peak shows shorter displacements after sloughing indicating reduced biomass and increase in pore spaces which allow flow at fixed volumetric flow rate.

Figure 4 shows detailed analysis of the profiles of $T_{2}$ distributions at zero displacement $Z=0$ and of the propagators at two specific $T_{2}$ values corresponding to bulk fluid $\left(T_{2} \sim 700 \mathrm{~ms}\right)$ and biomass $\left(T_{2} \sim 150 \mathrm{~ms}\right)$. Further evidence of a sloughing event occurring around $30-33 \mathrm{~h}$ of biofilm growth is shown in the decrease in the signal amplitude of the shorter $T_{2}$ peak between the second and third rows in Figure 4b. The propagator at the longer $T_{2}$ of approximately $700 \mathrm{~ms}$ corresponds to the flow of the bulk fluid and is not shifted significantly from the initial value of the $T_{2}$ of the BHI growth media. As the biofilm grew and plugged some of the pores of the bead pack, the distributions of displacements include longer displacements. This indicates a channeling effect, where the bulk fluid is not flowing through the biofilm-clogged pores and as a result is flowing faster through the clear pores to maintain the conservation of mass at the set volumetric flow rate (Seymour et al., 2004b). At the shorter $T_{2}$ of $\sim 150 \mathrm{~ms}$ that corresponds to the biofilm, there is no flow and the propagator is narrow and centered at $Z=0$. This is quantitative experimental evidence that there is no convective flow through the biomass within the displacement resolution of the measurement of $\pm 15.6 \mu \mathrm{m}$ over $\Delta=250 \mathrm{~ms}$, or $v= \pm 62 \mu \mathrm{m} / \mathrm{s}$.

Figure 5 presents an alternative analysis of the difference between the dynamics in the bulk fluid and within the
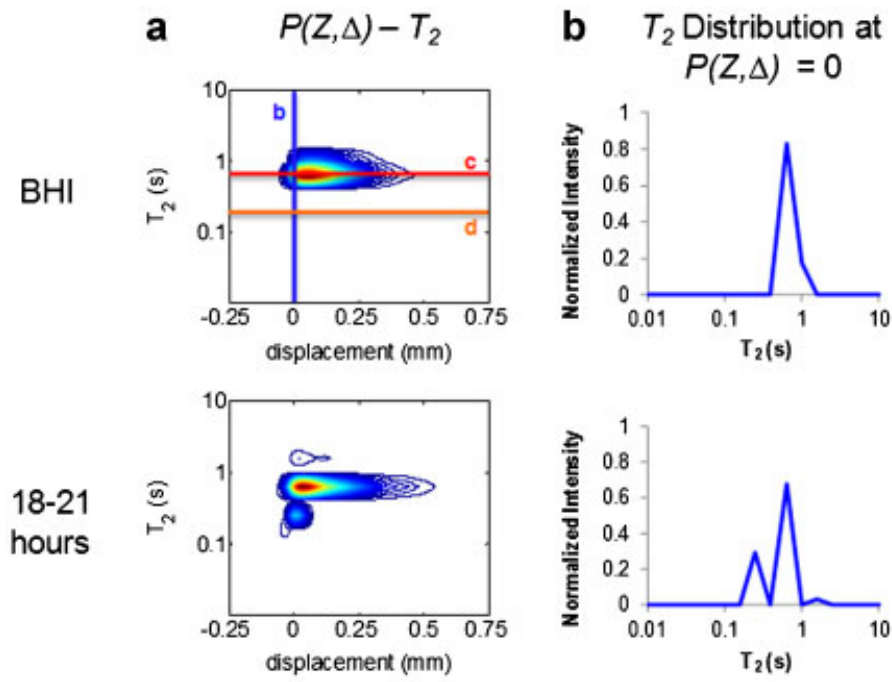

18-21

hours
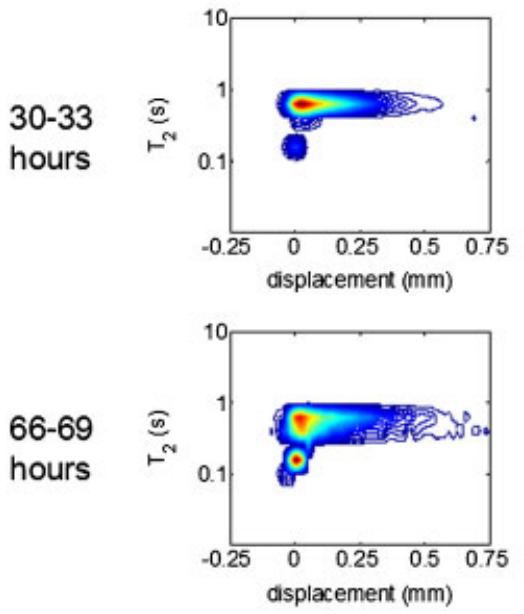
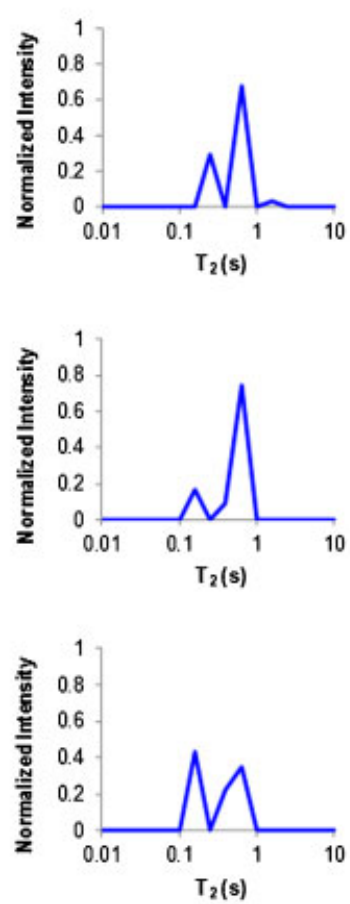
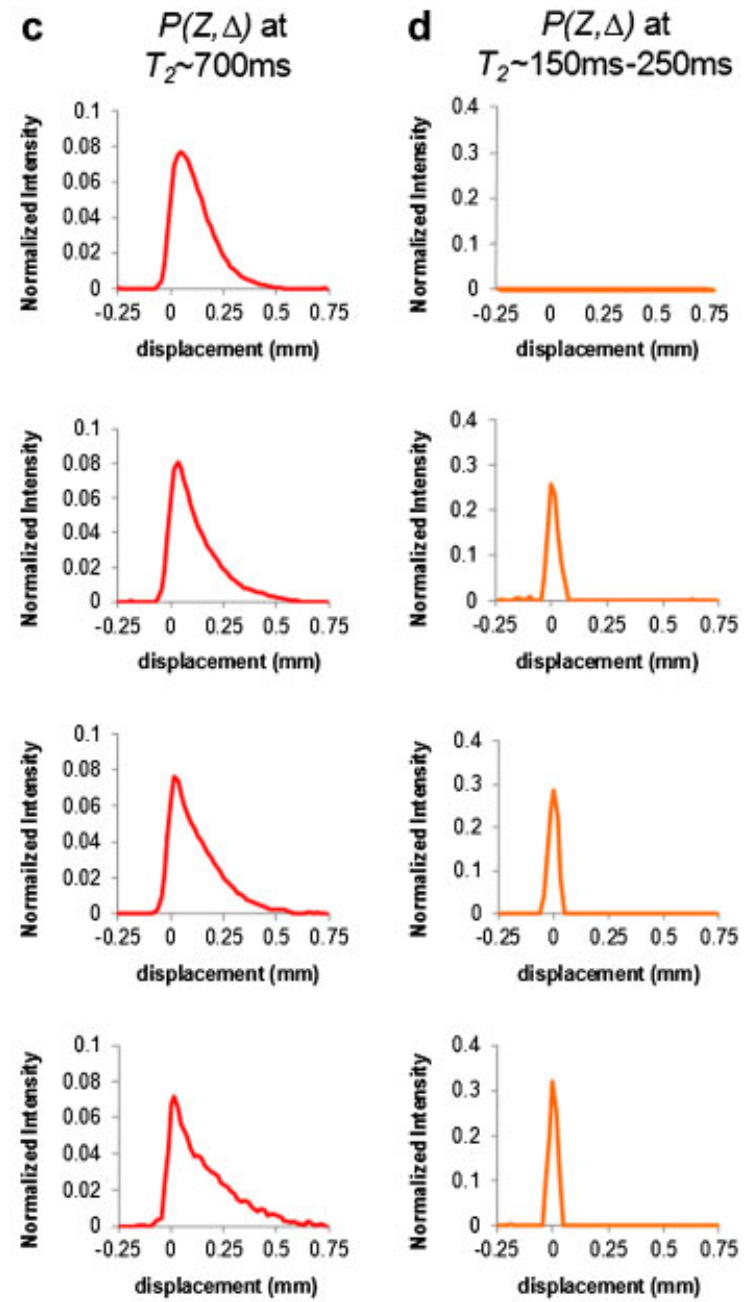

Figure 4. $T_{2}$ and $P(Z, \Delta)$ profiles at selected biofilm growth times for $\Delta$ of $250 \mathrm{~ms}$. a: $P(Z, \Delta)-T_{2}$ plots at three time points in the biofilm growth. b: $T_{2}$ profile taken at the $Z=0$ displacement, showing the growth in the shorter $T_{2}$ peak over time. c: $P(Z, \Delta)$ profile through the longer $T_{2}$ value, demonstrating that bulk fluid exhibits longer displacement values as the biofilm plugs some of the pores of the porous media. d: $P(Z, \Delta)$ profile through the shorter $T_{2}$ value, integrated between 150 and $250 \mathrm{~ms}$ in the $T_{2}$ direction, demonstrating that there is zero flow through the biomass. 


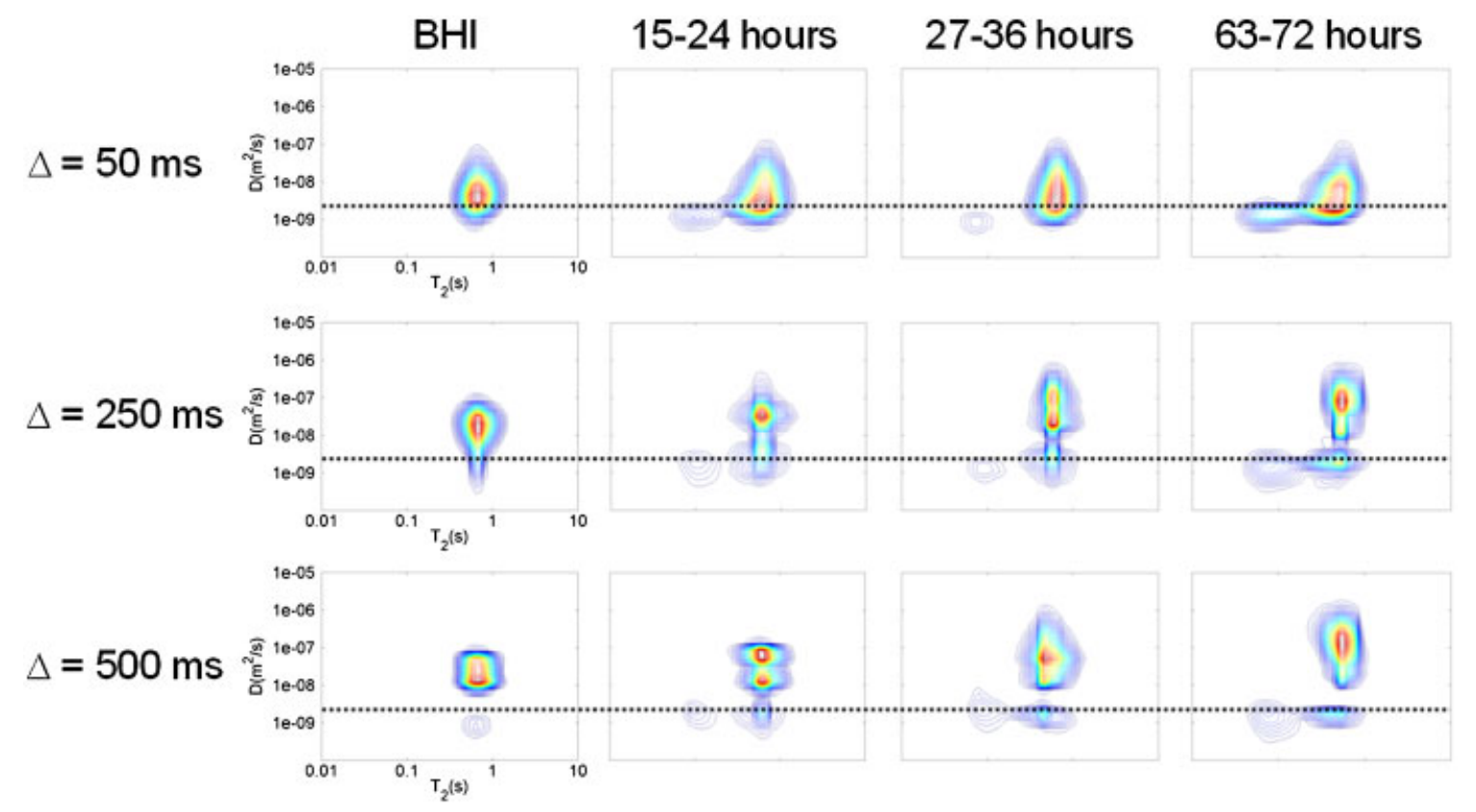

Figure 5. $D(\Delta)-T_{2}$ effective diffusion-spin-spin relaxation correlation plots of longitudinal hydrodynamic dispersion and fluid type for three different observation times and at four different times during biofilm growth. The dotted lines are shown as a guide to the eye at the self-diffusion of water at $25 \mathrm{C}, D_{0}=2.2 \times 10^{-9} \mathrm{~m}^{2} / \mathrm{s}$. Note the presence of a bimodal distribution in hydrodynamic dispersion at $\Delta=500 \mathrm{~ms}$ before the inoculation of bacteria. As the biofilm grew, the $\Delta=50 \mathrm{~ms}$ data continue to show about the same amount of hydrodynamic dispersion at $T_{2} \sim 700 \mathrm{~ms}$, while the 250 and $500 \mathrm{~ms}$ data show larger amplitude hydrodynamic dispersion more broadly distributed in amplitude as the biofilm grew. The spread in the vertical direction indicates the increase in hydrodynamic dispersion over biofilm growth for the bulk fluid indicating increased hydrodynamic mixing. The bimodal distribution of dispersion at $T_{2} \sim 700 \mathrm{~ms}$ also indicates the presence of dead end pores due to bioclogging. The spins at the shorter $T_{2}$ values however show no hydrodynamic dispersion on these time scales, indicating that there is no convective motion through the biomass.

biomass. Rather than performing a Fourier transform on the data, the positive pulsed magnetic field gradient displacement data was fitted with a $2 \mathrm{D}$ inverse Laplace transform corresponding to Equation (7) in the $q$-space direction and an exponential decay in the $T_{2}$ direction. These $2 \mathrm{D}$ plots are correlations between longitudinal hydrodynamic dispersion coefficients $D(\Delta)\left(\mathrm{m}^{2} / \mathrm{s}\right)$ along the vertical axis and magnetic spin-spin relaxation $T_{2}(\mathrm{~s})$ along the horizontal axis. The absolute value of the data is analyzed so all phase information is lost and instead of a phase shift corresponding to the velocity induced displacement, only hydrodynamic dispersion information is retained (Seymour and Callaghan, 1997). Before the bacteria were inoculated into the system, the results for the three displacement observation times $\Delta$ show the well-known increase in preasymptotic hydrodynamic dispersion $D(\Delta$; Codd and Seymour, 2012) and the same $T_{2}$ of $700 \mathrm{~ms}$. As the biofilm grew, the $\Delta=50 \mathrm{~ms}$ data continue to show about the same amount of hydrodynamic dispersion at $T_{2} \sim 700 \mathrm{~ms}$, while the 250 and $500 \mathrm{~ms}$ data show larger amplitude hydrodynamic dispersion more broadly distributed in amplitude as the biofilm grew. The decrease in signal intensity of the shorter $T_{2}$ peak in the 50 and $250 \mathrm{~ms}$ data is evident between the second and third columns, again demonstrating that a sloughing event occurred at about $30 \mathrm{~h}$ of biofilm growth. At these flow rates and $\Delta$ times, the protons on the water molecules have transited only $\left\langle Z^{2}(\Delta)\right\rangle^{1 / 2}=\left\langle\xi_{0}\right\rangle=\left\langle v_{0}\right\rangle \Delta=27,130$, and $270 \mu \mathrm{m}$ for $\Delta=50,250$, and $500 \mathrm{~ms}$ respectively, which provides displacement data in the clean bead pack ranging from within a single pore to several pores for the pore length scale $l=180 \mu \mathrm{m}$. The increasing amount of dispersion is a result of the growth of biofilm in parts of the bead pack and channeling in other parts of the bead pack causing increased mixing and dispersion. In contrast, at all biofilm growth times and all three $\Delta$ times, the peak at the $T_{2} \sim 150 \mathrm{~ms}$ corresponding to the biofilm shows no increased dispersion and is approximately along the line for the self-diffusion of water, again indicating that there is no convective flow generating dispersion within the biomass. An interesting feature of the data in Figure 5 is the evolution as a function of displacement time of a bimodal distribution of hydrodynamic dispersion in the BHI at the bulk fluid $T_{2} \sim 700 \mathrm{~ms}$. At displacement time $\Delta=500 \mathrm{~ms}$, for which $\left\langle\xi_{0}\right\rangle=270 \mu \mathrm{m}$, the flowing fluid has a mean dispersion of $D \sim 5 \times 10^{-8} \mathrm{~m}^{2} / \mathrm{s}$ while a smaller percentage of fluid has a value slightly slower than free water in BHI due to the no slip zero velocity layer at the bead surfaces. This restricted diffusion bulk fluid peak increases in concert with the biofilm peak at $T_{2} \sim 150 \mathrm{~ms}$ indicating increased hold-up of the bulk fluid contained in pore centers of biofouled pores and in dead end pores blocked by biofouled pores downstream. In addition, exchange occurring between the 


\section{a $12.5 \%$}

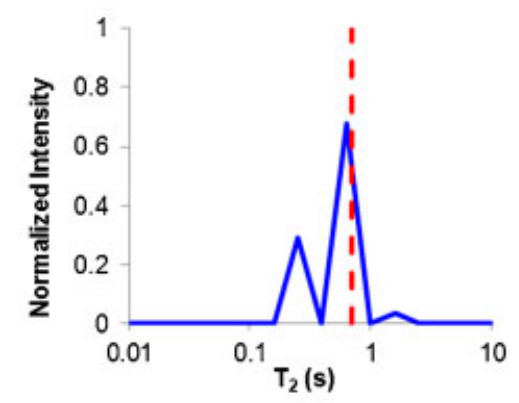

b $25 \%$

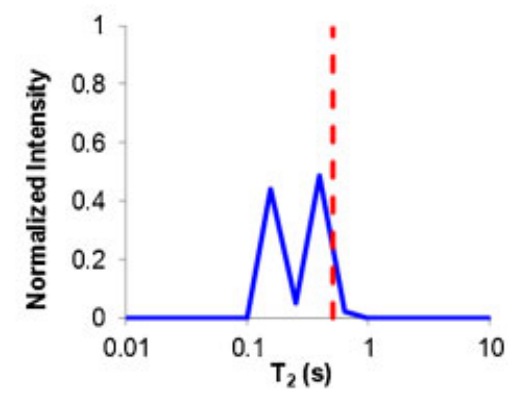

c $50 \%$

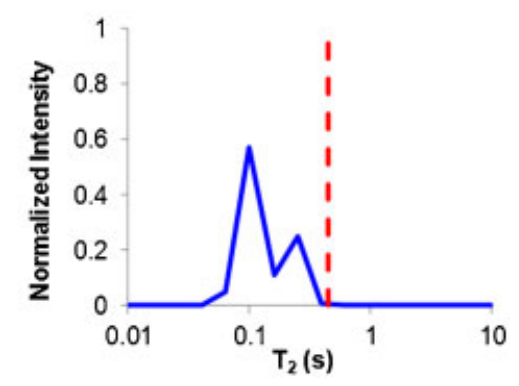

d $65 \%$

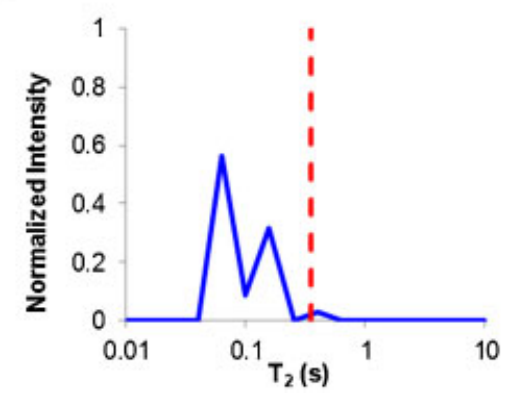

Figure 6. $T_{2}$ distributions at $P(Z, \Delta)=0$ and $18-21 \mathrm{~h}$ of biofilm growth for four different strengths of growth media: (a) $12.5 \%$, (b) $25 \%$, (c) $50 \%$, and (d) $65 \%$. The dotted lines indicate the $T_{2}$ of the growth media before biofilm growth: $690 \mathrm{~ms}$ for $12.5 \% \mathrm{BHI}$, $520 \mathrm{~ms}$ for $25 \% \mathrm{BHI}, 440 \mathrm{~ms}$ for $50 \% \mathrm{BHI}$, and $350 \mathrm{~ms}$ for $65 \% \mathrm{BHI}$. The $\mathrm{T}_{2}$ in the $\mathrm{BHI}$ is affected by the concentration of the carbohydrates and proteins in the growth media.

biofilm phase and the bulk phase in dead end pores may contribute to the increase in amplitude of the zero flow bulk fluid peak in the $D(\Delta)-T_{2}$ spectrum. This effect may be seen in the data for $63-72 \mathrm{~h}$, which shows a range of $T_{2}$ values with restricted diffusion. Hydrodynamic dispersion on the pore scale will impact Darcy scale reactive transport simulations and measurements (Battiato et al., 2009), so this ability to non-invasively measure dispersion of the biofilm phase and bulk fluid phase on the pore scale may provide data useful for a wide range of applications.

Figure 6 presents the $T_{2}$ distributions at $P(Z, \Delta)=0$ for $\Delta=250 \mathrm{~ms}$ and $21 \mathrm{~h}$ of biofilm growth for four different strengths of BHI. The dotted lines indicate the $T_{2}$ of the BHI before biofilm growth with decreasing $T_{2}=690,520$, 440 , and $350 \mathrm{~ms}$ for $12.5 \%, 25 \%, 50 \%$, and $65 \% \mathrm{BHI}$, respectively. The $T_{2}$ of all of the water in the bead pack is shown to shift to shorter times for all BHI strengths because the bulk fluid interacts with the polymers in the EPS of the biofilm and the $T_{2}$ is shifted due to hydrogen exchange (Hills et al., 1991). The ratio between the amplitudes of the two peaks changes for the larger strengths of BHI indicating that more biofilm has grown in the bead pack. By providing more nutrient to the system by increasing the strength of BHI fed to the system, more bacteria will reproduce and grow within the biofilm and produce EPS, leading to a denser polymer matrix which will decrease the $T_{2}$ (Fabich et al., 2012). In addition the entire $T_{2}$ distribution shifts to shorter $T_{2}$ values for increased biofilm growth, again indicating that the water interacts with more polymers in the biofilm, providing an additional measure for the extent of biofouling.

\section{Conclusion}

In this article, MR displacement-relaxation correlation experiments are demonstrated to characterize fluid transport in biofouled porous media by separate measurement of purely diffusive transport in the biofilm biomass and convection through the channels of the porous media. Within the displacement resolution of these experiments, no convective flow was measured through the biomass. An increased amount of longitudinal hydrodynamic dispersion indicates increased hydrodynamic mixing due to increased channeling effects caused by biofilm growth. Increasing the velocity resolution of the experiments is possible by increasing the number of displacement encoding steps but comes at the expense of longer experiments, resulting in data with high velocity resolution but very course resolution in the time of biofilm growth. Studies with different flow rates, different geometries, or different organisms have the potential to yield relevant data for the understanding of biofilm growth, flow dynamics, and hydrodynamic dispersion in porous media.

The authors would like to thank the late Prof. Sir Paul T. Callaghan for the inverse Laplace transform software and intellectual support.

\section{References}

Abragam A. 1961. The principles of nuclear magnetism. Oxford: Clarendon Press.

Bartacek J, Vergeldt FJ, Gerkema E, Jenicek P, Lens PNL, Van As H. 2009. Magnetic resonance microscopy of iron transport in methanogenic granules. J Magn Reson 200(2):303-312. 
Battiato I, Tartakovsky DM, Tartakovsky AM, Scheibe T. 2009. On breakdown of macroscopic models of mixing-controlled heterogeneous reactions in porous media. Adv Water Resour 32(11):16641673.

Beveridge TJ, Makin SA, Kadurugamuwa JL, Li ZS. 1997. Interactions between biofilms and the environment. Fems Microbiol Rev 20(34):291-303.

Brinkman HC. 1947. A calculation of the viscous force exerted by a flowing fluid on a dense swarm of particles. Appl Sci Res A Mech Heat Chem Eng Math Methods 1(1):27-34.

Britton MM, Graham RG, Packer KJ. 2004. NMR relaxation and pulsed field gradient study of alginate bead porous media. J Magn Reson 169(2):203-214.

Burcaw LM, Hunter MW, Callaghan PT. 2010. Propagator-resolved 2D exchange in porous media in the inhomogeneous magnetic field. J Magn Reson 205(2):209-215.

Callaghan PT. 1991. Principles of nuclear magnetic resonance microscopy. Oxford: Clarendon Press.

Callaghan PT. 2011. Translational dynamics and magnetic resonance: Principles of pulsed gradient spin echo NMR. Oxford: Oxford University Press.

Callaghan PT, Codd SL, Seymour JD. 1999. Spatial coherence phenomena arising from translational spin motion in gradient spin echo experiments. Concepts Magn Reson 11(4):181-202.

Carr HY, Purcell EM. 1954. Effects of diffusion on free precession in nuclear magnetic resonance experiments. Phys Rev 94(3):630-638.

Carver JP, Richards RE. 1972. General 2-site solution for chemical exchange produced dependence of $T_{2}$ upon Carr-Purcell pulse separation. J Magn Reson 6(1):89-105.

Codd SL, Seymour JD. 2012. Nuclear magnetic resonance measurement of hydrodynamic dispersion in porous media: Preasymptotic dynamics, structure and nonequilibrium statistical mechanics. Eur Phys J Appl Phys 60(2):24204-1-9.

Codd SL, Vogt SJ, Hornemann JA, Phillips AJ, Maneval JE, Romanenko KR, Hansen L, Cunningham AB, Seymour JD. 2011. NMR relaxation measurements of biofouling in model and geological porous media. Org Geochem 42(8):965-971.

Costerton JW, Stewart PS, Greenberg EP. 1999. Bacterial biofilms: A common cause of persistent infections. Science 284(5418):13181322.

Dupin HJ, Kitanidis PK, McCarty PL. 2001. Pore-scale modeling of biological clogging due to aggregate expansion: A material mechanics approach. Water Resour Res 37(12):2965-2979.

Fabich HT, Vogt SJ, Sherick ML, Seymour JD, Brown JR, Franklin MJ, Codd SL. 2012. Microbial and algal alginate gelation characterized by magnetic resonance. J Biotechnol 161(3):320-327.

Fang YL, Yabusaki SB, Morrison SJ, Amonette JP, Long PE. 2009. Multicomponent reactive transport modeling of uranium bioremediation field experiments. Geochimica Et Cosmochimica Acta 73(20):60296051.

Hills BP, Cano C, Belton PS. 1991. Proton Nmr relaxation studies of aqueous polysaccharide systems. Macromolecules 24(10):29442950.

Homan NM, Venne B, Van As H. 2010. Flow characteristics and exchange in complex biological systems as observed by pulsed-field-gradient magnetic-resonance imaging. Phys Rev E 82(2):026310-1-9.

Hornemann JA, Lysova AA, Codd SL, Seymour JD, Busse SC, Stewart PS, Brown JR. 2008. Biopolymer and water dynamics in microbial biofilm extracellular polymeric substance. Biomacromolecules 9(9):2322-2328.

Hoskins BC, Fevang L, Majors PD, Sharma MM, Georgiou G. 1999. Selective imaging of biofilms in porous media by NMR relaxation. J Magn Reson 139(1):67-73.

Kapellos GE, Alexiou TS, Payatakes AC. 2007. Hierarchical simulator of biofilm growth and dynamics in granular porous materials. Adv Water Resour 30(6-7):1648-1667.

Kim DS, Fogler HS. 2000. Biomass evolution in porous media and its effects on permeability under starvation conditions. Biotechnol Bioeng 69(1):47-56.
Klapper I, Rupp CJ, Cargo R, Purvedorj B, Stoodley P. 2002. Viscoelastic fluid description of bacterial biofilm material properties. Biotechnol Bioeng 80(3):289-296.

Lens P, Pol LH, Lettinga G, Van As H. 1997. Use of H-1 NMR to study transport processes in sulfidogenic granular sludge. Water Sci Technol 36(6-7):157-163.

Lens P, Vergeldt F, Lettinga G, Van As H. 1999. H-1 NMR characterisation of the diffusional properties of methanogenic granular sludge. Water Sci Technol 39(7):187-194.

Manz B, Volke F, Goll D, Horn H. 2003. Measuring local flow velocities and biofilm structure in biofilm systems with magnetic resonance imaging (MRI). Biotechnol Bioeng 84(4):424-432.

McLean JS, Majors PD, Reardon CL, Bilskis CL, Reed SB, Romine MF, Fredrickson JK. 2008. Investigations of structure and metabolism within Shewanella oneidensis MR-1 biofilms. J Microbiol Methods 74(1):47-56.

Mitchell AC, Phillips AJ, Hamilton MA, Gerlach R, Hollis WK, Kaszuba JP, Cunningham AB. 2008a. Resilience of planktonic and biofilm cultures to supercritical CO2. J Supercrit Fluids 47(2):318-325.

Mitchell J, von der Schulenburg DAG, Holland DJ, Fordham EJ, Johns ML, Gladden LF. 2008b. Determining NMR flow propagator moments in porous rocks without the influence of relaxation. J Magn Reson 193(2):218-225.

Picioreanu C, van Loosdrecht MCM, Heijnen JJ. 2001. Two-dimensional model of biofilm detachment caused by internal stress from liquid flow. Biotechnol Bioeng 72(2):205-218.

Pintelon TRR, Creber SA, von der Schulenburg DAG, Johns ML. 2010. Validation of 3D simulations of reverse osmosis membrane biofouling. Biotechnol Bioeng 106(4):677-689.

Pintelon TRR, Picioreanu C, van Loosdrecht MCM, Johns ML. 2012. The effect of biofilm permeability on bio-clogging of porous media. Biotechnol Bioeng 109(4):1031-1042.

Radu AI, Vrouwenvelder JS, van Loosdrecht MCM, Picioreanu C. 2012. Effect of flow velocity, substrate concentration and hydraulic cleaning on biofouling of reverse osmosis feed channels. Chem Eng J 188:30-39.

Renslow RS, Majors PD, McLean JS, Fredrickson JK, Ahmed B, Beyenal H. 2010. In situ effective diffusion coefficient profiles in live biofilms using pulsed-field gradient nuclear magnetic resonance. Biotechnol Bioeng 106(6):928-937.

Seymour JD, Callaghan PT. 1997. Generalized approach to NMR analysis of flow and dispersion in porous media. Aiche J 43(8):2096-2111.

Seymour JD, Codd SL, Gjersing EL, Stewart PS. 2004a. Magnetic resonance microscopy of biofilm structure and impact on transport in a capillary bioreactor. J Magn Reson 167(2):322-327.

Seymour JD, Gage JP, Codd SL, Gerlach R. 2004b. Anomalous fluid transport in porous media induced by biofilm growth. Phys Rev Lett 93(19):198103-1-4.

Seymour JD, Gage JP, Codd SL, Gerlach R. 2007. Magnetic resonance microscopy of biofouling induced scale dependent transport in porous media. Adv Water Resour 30(6-7):1408-1420.

Stejskal EO, Tanner JE. 1965. Spin diffusion measurements: Spin echoes in the presence of a time-dependent field gradient. J Chem Phys 42(1):288-292.

Stewart PS. 2012. Mini-review: Convection around biofilms. Biofouling 28(2):187-198.

Sutherland IW. 2001. Biofilm exopolysaccharides: A strong and sticky framework. Microbiology-UK 147:3-9.

Thullner M, Baveye P. 2008. Computational pore network modeling of the influence of biofilm permeability on bioclogging in porous media. Biotechnol Bioeng 99(6):1337-1351.

Venkataramanan L, Song Y-Q, Hurlimann MD. 2002. Solving fredholm integrals of the first kind with tensor product structure in 2 and 2.5 dimensions. IEEE Trans Signal Process 50(5):1017-1026.

von der Schulenburg DAG, Holland DJ, Paterson-Beedle M, Macaskie LE, Gladden LF, Johns ML. 2008a. Spatially resolved quantification of metal ion concentration in a biofilm-mediated ion exchanger. Biotechnol Bioeng 99(4):821-829. 
von der Schulenburg DAG, Vrouwenvelder JS, Creber SA, van Loosdrecht MCM, Johns ML. 2008b. Nuclear magnetic resonance microscopy studies of membrane biofouling. J Membr Sci 323(1):37-44.

von der Schulenburg DAG, Pintelon TRR, Picioreanu C, Van Loosdrecht MCM, Johns ML. 2009. Three-dimensional simulations of biofilm growth in porous media. Aiche J 55(2):494-504.
Washburn KE, Callaghan PT. 2007. Propagator resolved transverse relaxation exchange spectroscopy. J Magn Reson 186(2):337340 .

Windt CW, Vergeldt FJ, Van As H. 2007. Correlated displacement-T-2 MRI by means of a Pulsed Field Gradient-Multi Spin Echo method. J Magn Reson 185(2):230-239. 